\title{
MODIFYING EIGENVALUE INTERACTIONS NEAR WEAK RESONANCE
}

\author{
Vincent Auvray* Ian Dobson \\ ECE Dept., University of Wisconsin \\ 1415 Engineering Drive \\ Madison WI 53706 USA \\ dobson@engr.wisc.edu
}

\author{
Louis Wehenkel \\ EE\&CS Dept., Université de Liège \\ Sart-Tilman B28 \\ B-4000 Liège, Belgique \\ L.Wehenkel@ulg.ac.be
}

\begin{abstract}
In electric power system instabilities such as subsynchronous resonance or interarea oscillations, two complex modes can approach each other in frequency and then interact by changing damping so that one of the modes becomes unstable. Selecting changes in parameters to minimize this interaction is difficult by trial and error. By analyzing the interaction as a perturbation of a weak resonance, we calculate sensitivities that indicate the parameters to be changed to minimize the interaction and stabilize the system. The method is illustrated with a simple example of two coupled linear oscillators. The use of sensitivity methods to change the type of the interaction is also demonstrated.
\end{abstract}

\section{INTRODUCTION}

A power system mode is an eigenvalue of the linearized system and its associated eigenvector. Since the modes determine the power system stability and small signal transient performance, understanding the behavior of the complex modes is fundamental to avoiding power system oscillations $[9,2,8]$. Indeed, a better understanding and suppression of low frequency interarea oscillations or subsynchronous resonance could relax some of the constraints on larger bulk power transfers and increase power system security.

An exact coincidence of complex eigenvalues in both frequency and damping is called a resonance. There are two types of resonance: strong and weak [3]. Strong resonance has a nondiagonalizable linearization and weak resonance has a diagonalizable linearization. As parameters vary, the power system modes vary and it is not uncommon for two complex eigenvalues to pass near resonance. For a comprehensive review up to 2000 see [3] and for more recent work see $[6,7,4,5]$. Only a subset of oscillatory power system problems involve resonant eigenvalues and there are

\footnotetext{
* Vincent Auvray is a visiting scholar at the University of Wisconsin and a F.N.R.S. research fellow at the University of Liège, Belgium. Funding in part from the F.N.R.S., Belgium, the University of Liège, and the National Science Foundation, USA under grant ECS-9988574 is gratefully acknowledged.
}

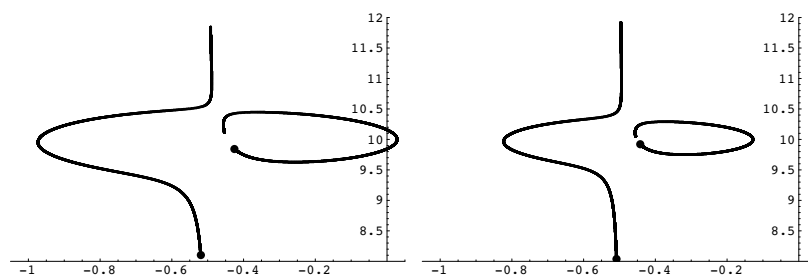

(a)

(b)

Fig. 1. Eigenvalue interactions as a parameter $t$ is varied. The dots indicate the initial eigenvalue positions. (a) shows eigenvalues initially moving together in frequency interacting by changes in damping so that one eigenvalue becomes unstable. (b) shows a minimized eigenvalue interaction that retains stability.

some established and useful mitigation methods for subsynchronous resonance and interarea oscillations.

Near a weak resonance, as a parameter $t$ is changed, two eigenvalues initially approaching each other in frequency can interact and move in damping so that one of the eigenvalues becomes unstable as shown in Figure 1(a). Similar interactions cause subsynchronous resonance instabilities [4] and instabilities in models of interarea oscillations [5]. Let $\delta$ be the maximum separation between the eigenvalues in damping (maximum difference in their real parts) as the parameter $t$ is changed. In this paper we compute the sensitivity of $\delta$ with respect to other parameters of the problem so that changes in these other parameters can be made to reduce $\delta$, so as to minimize the interaction and stabilize the system as shown in Figure 1(b). This systematic procedure to minimize the interaction does not require insight into the relatively complicated eigenvalue movements near weak resonance and their relation to the parameters of the problem, nor does it require exhaustively recomputing the root locus for each parameter by trial and error. 


\section{INTERACTION NEAR A WEAK RESONANCE}

The power system differential equations are assumed to depend smoothly on a real parameter $t$ that parameterizes the eigenvalue movement in the complex plane. That is, $t$ is the root locus parameter. The system Jacobian evaluated at the operating equilibrium is the real matrix $J(t)$ and has two complex eigenvalues of interest $\lambda_{1}(t)$ and $\lambda_{2}(t)$.

The eigenvalues do not vary smoothly at weak resonance. However, the following function can be shown to smoothly depend on the parameters $[5,3]$ :

$$
\mu(t)=\left(\lambda_{1}(t)-\lambda_{2}(t)\right)^{2} / 4 .
$$

$\mu$ determines the separation of the eigenvalues in both magnitude and angle. That is, $\mu$ determines the relative position of the eigenvalues. The distance between the eigenvalues is $\left|\lambda_{1}-\lambda_{2}\right|=2 \sqrt{|\mu|}$. In particular, the condition for coincident and resonant eigenvalues is $\mu=0$. Moreover, the relative direction of the eigenvalues is $\angle\left(\lambda_{1}-\lambda_{2}\right)=\frac{1}{2} \angle \mu+k \pi$, for some integer $k$. Dobson [5] shows that close to a weak resonance, the locus $t \mapsto \mu(t)$ for real $t$ describes to second order a parabola in the complex plane passing near the origin. In this paper, we propose a method to modify the eigenvalue movement.

\section{STABILIZING THE INTERACTION}

We first introduce a measure of the interaction of the eigenvalues near weak resonance. Suppose that the two eigenvalues resonate weakly for some $t$, say $t=0$. Dobson [5] shows that this implies $\mu_{t}(0)=\mu(0)=0$, where the subscript denotes differentiation. A weak resonance is thus a root of $\mu$ of multiplicity at least two. We make the generic assumption that $A=\frac{1}{2} \mu_{t t}(0) \neq 0$; i.e., $t=0$ is a double root. A Taylor expansion around $t=0$ gives

$$
\mu(t)=A t^{2}+o\left(|t|^{2}\right) .
$$

Equation (2) implies that the eigenvalues approach each other along a line of angle $\theta=\frac{1}{2} \angle A$ as $t \rightarrow 0$.

To model the proximity to a weak resonance, the Jacobian is supposed to depend smoothly on an additional parameter $\epsilon$ such that there is weak resonance at $(t, \epsilon)=$ $(0,0)$. For small fixed $\epsilon \neq 0$, the eigenvalues pass close to weak resonance as $t$ passes through 0 . Define $d$ as the length of the projection of $\lambda_{1}-\lambda_{2}$ on the direction perpendicular to $\theta$. For $\theta=\frac{\pi}{2}, d$ is the size of the difference in damping between the eigenvalues. We have

$$
\begin{aligned}
d & =\left|\lambda_{1}-\lambda_{2}\right|\left|\sin \left(\angle\left(\lambda_{1}-\lambda_{2}\right)-\theta\right)\right| \\
& =2 \sqrt{|\mu|}\left|\sin \left(\frac{1}{2} \angle \mu-\theta\right)\right| .
\end{aligned}
$$

Equation (4) shows that $d(t)$ can be defined as a function $d(\mu(t))$ of $\mu$ alone. One can see that $d(\mu) \leq K$ if, and only if, in the complex plane the point $\mu e^{-2 i \theta}$ is contained inside the parabola parametrized by

$$
\nu(s)=\left(s^{2}-\frac{K^{2}}{4}\right)+i K s, \quad s \in \mathbb{R} .
$$

We propose to measure the extent of the eigenvalue interaction by

$$
\delta(\eta, \epsilon)=\max _{|t| \leq \eta} d(\mu(t, \epsilon)),
$$

where $\eta>0$.

Let us construct an approximation $\mu^{a}$ of $\mu$ that will allow us to modify the shape of the locus of $\mu$ and, in particular, influence the quantity (6). The system Jacobian evaluated at the operating equilibrium is supposed to depend on a vector of parameters $p=\left(p_{1}, p_{2}, \ldots, p_{m}\right)$ that can be varied to modify the eigenvalue interaction. The nominal parameter vector is $p=0$ and the eigenvalues resonate weakly for $(t, \epsilon, p)=(0,0,0)$.

One key mathematical step is to consider the variables $t$, $\epsilon$ and $p$ as complex. Recall that for $(\epsilon, p)=(0,0), t=0$ is a real double root of $\mu$. Let us show that for small $(\epsilon, p)$, this root generically perturbs to two complex single roots $r_{1}$ and $r_{2}$. We suppose that $J$, and thus $\mu$, is analytic in $t, \epsilon$ and $p$. It follows from the Weierstrass preparation theorem [1] that there exist unique continuous functions $r_{1}(\epsilon, p)$ and $r_{2}(\epsilon, p)$ defined for $(\epsilon, p)$ in a sufficiently small neighborhood of $(0,0)$ and such that $r_{i}(0,0)=0, \mu\left(r_{i}(\epsilon, p), \epsilon, p\right)=0$ and $r_{i}$ is analytic at the points $(\epsilon, p)$ where $r_{1} \neq r_{2}$. We thus generically suppose that $r_{1}$ and $r_{2}$ are distinct at the points considered.

Define the following approximation to $\mu$

$$
\mu^{a}(t, \epsilon, p)=A\left(t-r_{1}(\epsilon, p)\right)\left(t-r_{2}(\epsilon, p)\right) .
$$

For real $t, \mu^{a}(t, \epsilon, p)$ traces a parabola in the complex plane as $t$ varies. The approximation (7) can be intuitively justified by the following reasoning. In a neighborhood of $(0,0,0), \mu$ can be expanded as

$$
\begin{aligned}
\mu(t, \epsilon, p) & =A t^{2}+2 B(\epsilon, p) t+C(\epsilon, p)+o\left(|(t, \epsilon, p)|^{2}\right)(8) \\
& =A\left(t-q_{1}(\epsilon, p)\right)\left(t-q_{2}(\epsilon, p)\right)+o\left(|(t, \epsilon, p)|^{2}\right),
\end{aligned}
$$

where $q_{1}, q_{2}=A^{-1}\left(-B \pm \sqrt{B^{2}-A C}\right)$ are the roots of the quadratic polynomial in $t$ of (8). To obtain $\mu^{a}$, we drop the high-order term $o\left(|(t, \epsilon, p)|^{2}\right)$ and replace $q_{1}$ and $q_{2}$ by $r_{1}$ and $r_{2}$.

Using approximation (7) we define

$$
\delta^{a}(\eta, \epsilon, p)=\max _{\{t|t \in \mathbb{R},| t \mid \leq \eta\}} d\left(\mu^{a}(t, \epsilon, p)\right)
$$

and for large enough $\eta$, evaluate this quantity with geometric relation (5) as

$$
\delta^{a}(\eta, \epsilon, p)=\sqrt{|A|} \sqrt{\left(\Re\left(r_{1}-r_{2}\right)\right)^{2}+\left(\Im\left(r_{1}+r_{2}\right)\right)^{2}} .
$$


We hence propose to use $\delta^{a}(\eta, \epsilon, p)$ and its vector of derivatives $\delta_{p}^{a}(\eta, \epsilon, p)=\left(\frac{\partial \delta^{a}}{\partial p_{1}}, \ldots, \frac{\partial \delta^{a}}{\partial p_{m}}\right)$ as approximations of $\delta(\eta, \epsilon, p)$ and $\delta_{p}(\eta, \epsilon, p)$ for small enough $p$.

$A, r_{1}(\epsilon, p)$ and $r_{2}(\epsilon, p)$ can be approximated by fitting a parabola to the observed $\mu$ locus for fixed $(\epsilon, p)$. Section 6 discusses the computation of the root sensitivities $r_{i p_{k}}=$ $\frac{\partial r_{i}}{\partial p_{k}}$. The sensitivity vector $\delta_{p}^{a}$ can be used to select and change parameters to stabilize the interaction, for example by a gradient descent incrementally minimizing $\delta^{a}$.

\section{EXAMPLE}

This section gives a simple example to illustrate the use of the sensitivities. We incrementally reduce $\delta$ to stabilize a system, changing the interaction of Figure 1(a) into the one in Figure 1(b).

Following [4], consider the 2 coupled linear oscillators

$$
\begin{gathered}
\ddot{x}+\dot{x}+t^{2} x=p_{1} \dot{y}, \\
\ddot{y}+p_{3} \dot{y}+100 y=p_{2} \dot{x} .
\end{gathered}
$$

The parameters $p_{1}$ and $p_{2}$ control their coupling, while $p_{3}$ controls the damping of the second oscillator. In state space form, (11) and (12) can be written as

$$
\frac{d}{d t}\left(\begin{array}{l}
x \\
\dot{x} \\
y \\
\dot{y}
\end{array}\right)=\left(\begin{array}{cccc}
0 & 1 & 0 & 0 \\
-t^{2} & -1 & 0 & p_{1} \\
0 & 0 & 0 & 1 \\
0 & p_{2} & -100 & p_{3}
\end{array}\right)\left(\begin{array}{l}
x \\
\dot{x} \\
y \\
\dot{y}
\end{array}\right) .
$$

Suppose that initially $\left(p_{1}, p_{2}, p_{3}\right)=(1,1,0.89)$ and $t$ varies from 8 to 12 . The locus of the eigenvalues of positive frequency is numerically computed and shown in Figure 1(a). Using equation (1), we can plot $\mu$ and observe that it describes a parabola around the origin. This suggests that the eigenvalues are passing close to a weak resonance. By numerically fitting a parabola to this observed $\mu$, the roots $r_{1}$ and $r_{2}$ are found to be approximately $9.00448-$ $i 0.104793$ and $11.0016-i 0.00997545$.

Let us iteratively minimize $\delta^{a}$. The $n$th step of the optimization modifies the vector $p$ of parameters as follows

$$
p^{(n)}=p^{(n-1)}-c \delta_{p}^{a}\left(p^{(n-1)}\right)
$$

where $c$ is a constant step size. The eigenvalue loci for the first steps of this process are shown in Figure 2.

\section{CHANGING INTERACTION TYPE}

It is known that there are two types of perturbations from a weak resonance: type A and type B [5]. In type A the eigenvalues do not pass each other as the parameter is varied and in type B, the eigenvalues pass each other. Examples of type A and B interactions are shown in Figure 3. This

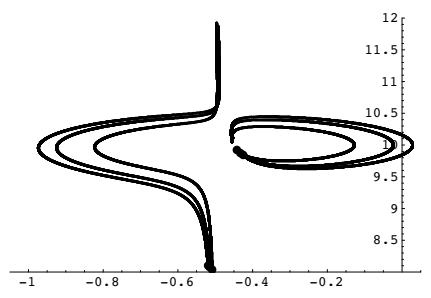

Fig. 2. First steps of gradient descent minimizing $\delta^{a}$.

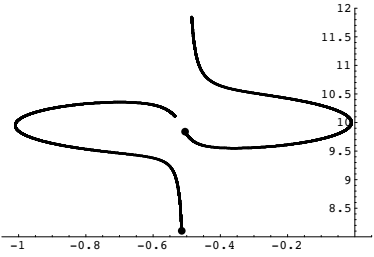

(a)

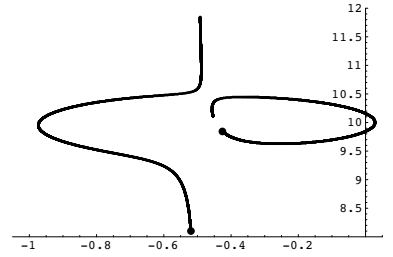

(b)
Fig. 3. Type A and type B eigenvalue interactions.

difference can be described in terms of the approximating parabola $\mu^{a}$. The interaction is type A if the parabola does not pass around the origin and type $\mathrm{B}$ if the parabola does pass around the origin [5].

We show how the roots $r_{1}$ and $r_{2}$ of (7) determine the type of interaction. Following [5], consider the function $f: \mathbb{R} \rightarrow \mathbb{C} ; k \mapsto A k$ that defines the line through the origin parallel to the principal axis of the parabola. The intersection of this line with the parabola generically occurs for $k=k^{*}$ and $t=t^{*}$. The eigenvalue interaction is type A if $k^{*}>0$, while it is type $\mathrm{B}$ if $k^{*}<0$. One can show that

$$
k^{*}=-\Im\left(r_{1}\right) \Im\left(r_{2}\right)\left[1+\left(\frac{\Re\left(r_{1}-r_{2}\right)}{\Im\left(r_{1}+r_{2}\right)}\right)^{2}\right] .
$$

Hence, the two eigenvalues have a type A or B interaction if $r_{1}$ and $r_{2}$ are, respectively, on opposite sides or the same side of the real axis.

The sensitivities $r_{i p_{k}}$ can be used to select parameters to move the roots $r_{1}, r_{2}$ from the same side of the real axis to opposite sides of the real axis, turning, for example, the type A interaction of Figure 3(a) to the type B interaction of Figure 3(b).

\section{THE ROOT SENSITIVITIES $r_{i p_{k}}$}

For notational convenience, we omit the dependence on $\epsilon$. Taking the derivative of $\mu\left(r_{i}(p), p\right)=0$ w.r.t. $p_{k}$ gives

$$
\mu_{t}\left(r_{i}(p), p\right) r_{i p_{k}}(p)+\mu_{p_{k}}\left(r_{i}(p), p\right)=0 .
$$


If we make the generic assumption that $\mu_{t}\left(r_{i}(p), p\right) \neq 0$, we have

$$
r_{i p_{k}}(p)=-\frac{\mu_{p_{k}}\left(r_{i}(p), p\right)}{\mu_{t}\left(r_{i}(p), p\right)} .
$$

Furthermore, the previous assumption that $r_{1}(p) \neq r_{2}(p)$ implies that $\left(r_{i}(p), p\right)$ are single roots and thus strong resonances.

Now we evaluate the sensitivity $\mu_{s}\left(s_{0}\right)$ where $\mu$ and $J$ are analytic functions of a complex parameter $s$ and where there is a strong resonance at $s_{0}$. Let $\lambda_{1}\left(s_{0}\right)=\lambda_{2}\left(s_{0}\right)=\lambda_{0}$ and let us make the generic assumption that the remaining eigenvalues $\lambda_{i}\left(s_{0}\right), i=3, \ldots, n$ are distinct. Let $v_{1}$ be a right eigenvector and $v_{1 g}$ a generalized right eigenvector of $J\left(s_{0}\right)$ related to $\lambda_{0}$ and let $v_{i}, i=3, \ldots, n$ be right eigenvectors of $J\left(s_{0}\right)$ related to, respectively, $\lambda_{i}\left(s_{0}\right)$, $i=3, \ldots, n$.

The theory of versal deformation presented in [10] states that there exists smooth matrices $C(s)$ and $V(s)$ defined in a neighborhood of $s_{0}$ such that

$$
J(s)=C(s) V(s)(C(s))^{-1} .
$$

$C(s)$ and $V(s)$ are not explicitly specified, but we know that $C(s)$ is invertible, $C\left(s_{0}\right)=\left(v_{1}, v_{1 g}, v_{3}, \ldots, v_{n}\right)$ and

$$
V(s)=\left(\begin{array}{ccccc}
\alpha(s) & 1 & & & \\
\mu(s) & \alpha(s) & & & \\
& & \lambda_{3}(s) & & \\
& & & \ddots & \\
& & & & \lambda_{n}(s)
\end{array}\right),
$$

where $\alpha\left(s_{0}\right)=\lambda_{0}$. One immediately notes that the first element of the second row is $\mu$. Also, recall that $\mu\left(s_{0}\right)=0$.

The left and right eigenvectors of $V\left(s_{0}\right)$ related to $\lambda_{0}$ are $l_{V\left(s_{0}\right)}=(0,1,0, \ldots, 0)$ and $r_{V\left(s_{0}\right)}=(1,0,0, \ldots, 0)^{\mathrm{T}}$. Hence, taking the derivative of (19),

$$
\begin{aligned}
\mu_{s}\left(s_{0}\right) & =l_{V\left(s_{0}\right)} V_{s}\left(s_{0}\right) r_{V\left(s_{0}\right)} \\
& =l_{V\left(s_{0}\right)}\left[C^{-1} J_{s} C+C^{-1} J C_{s}+\left(C^{-1}\right)_{s} J C\right] r_{V\left(s_{0}\right)} \\
& =l_{J\left(s_{0}\right)}\left[J_{s}+J C_{s} C^{-1}+C\left(C^{-1}\right)_{s} J\right] r_{J\left(s_{0}\right)} \\
& =l_{J\left(s_{0}\right)}\left[J_{s}+\lambda_{0} C_{s} C^{-1}+\lambda_{0} C\left(C^{-1}\right)_{s}\right] r_{J\left(s_{0}\right)} \\
& =l_{J\left(s_{0}\right)} J_{s}\left(s_{0}\right) r_{J\left(s_{0}\right)},
\end{aligned}
$$

where $l_{J\left(s_{0}\right)}$ and $r_{J\left(s_{0}\right)}$ are the left and right eigenvectors of $J\left(s_{0}\right)$ related to $\lambda_{0}$ obtained by transforming $l_{V\left(s_{0}\right)}$ and $r_{V\left(s_{0}\right)}$ according to

$l_{J\left(s_{0}\right)}=\left(C\left(s_{0}\right)\right)^{-1} l_{V\left(s_{0}\right)}$ and $r_{J\left(s_{0}\right)}=C\left(s_{0}\right) r_{V\left(s_{0}\right)}$.

Combining (17) and (20), we obtain

$$
r_{i p_{k}}(p)=-\frac{l_{i} J_{p_{k}}\left(r_{i}(p), p\right) r_{i}}{l_{i} J_{t}\left(r_{i}(p), p\right) r_{i}}, \quad i=1,2,
$$

where $l_{i}$ and $r_{i}$ are the eigenvectors of $J\left(r_{i}(p), p\right)$ with their scaling specified by (21). However, the scaling of the eigenvectors cancels in the quotient of (22). Hence, it is not necessary to compute $C\left(s_{0}\right)$. The eigenvectors can be computed from $J\left(r_{i}(p), p\right)$ with any scaling.

\section{CONCLUSION}

We have shown that the interaction between two eigenvalues as they pass near weak resonance is governed by two complex roots. (The two roots $r_{1}$ and $r_{2}$ are complex values of the root locus parameter that zero the square of the difference between the two interacting eigenvalues and that determine strong resonances.) Using quadratic approximations, we compute the sensitivity of these roots to parameter variations so that parameter changes to move the roots can be selected to minimize the interaction and also to change the interaction type. The method is illustrated in a simple example. Application to more realistic examples, particularly to minimize loss of damping due to eigenvalue resonance, is the next step of research.

\section{REFERENCES}

[1] S-N. Chow, J.K. Hale, Methods of bifurcation theory, Springer NY 1982.

[2] Cigré Task Force 07 of Advisory Group 01 of Study Committee 38, Analysis and control of power system oscillations, Paris, December 1996.

[3] I. Dobson, J. Zhang, S. Greene, H. Engdahl, P.W. Sauer, Is strong modal resonance a precursor to power system oscillations?, IEEE Trans. Circuits and Systems, Part 1, vol. 48, no. 3, March 2001, pp. 340-349.

[4] I. Dobson, Strong resonance effects in normal form analysis and subsynchronous resonance, Bulk Power System Dynamics and Control V, August 2001, Onomichi, Japan.

[5] I. Dobson, E. Barocio, Perturbations of weakly resonant power system electromechanical modes, Bologna Power Tech, Italy, June 2003.

[6] L. Jones, G. Andersson, V. Knyazkin, On modal resonance and interarea oscillations in power systems, Bulk Power System Dynamics and Control V, Aug. 2001, Onomichi, Japan.

[7] B.M. Nomikos, C.D. Vournas, Modal interaction and PSS design, Porto Power Tech Conference, Portugal, Sept. 2001.

[8] K.R. Padiyar, Analysis of Subsynchronous Resonance in Power Systems, ISBN 0792383192, Kluwer, 1998.

[9] G. Rogers, Power system oscillations, Kluwer, 2000.

[10] S. Wiggins, Introduction to applied nonlinear dynamical systems and chaos, Springer-Verlag, 1990. 